\title{
Pour une formation des élus municipaux en matière d'environnement : observations et repères
}

Marc-André Guertin

\section{(2) OpenEdition \\ Journals}

Édition électronique

URL : https://journals.openedition.org/ere/6299

DOI : $10.4000 /$ ere.6299

ISSN : 2561-2271

Éditeur

Centr'ERE

Référence électronique

Marc-André Guertin, « Pour une formation des élus municipaux en matière d'environnement :

observations et repères », Éducation relative à l'environnement [En ligne], Volume 16 - 1 | 2021, mis en ligne le 10 mars 2021, consulté le 28 mai 2021. URL : http://journals.openedition.org/ere/6299 ; DOI : https://doi.org/10.4000/ere.6299

Ce document a été généré automatiquement le 28 mai 2021. 


\title{
Pour une formation des élus municipaux en matière d'environnement : observations et repères
}

\author{
Marc-André Guertin
}

1 Comme en témoigne ce numéro thématique, définir ce qu'est un adulte dans toute la complexité et la diversité de ses rapports à l'environnement, pose un certain nombre de défis en éducation relative à l'environnement ( Lange, 2010a; Clover et Hill, 2013 ; Martin et Chen, 2016; Villemagne, 2008, 2017) et plus spécifiquement, en matière de formation spécialisée. Par ailleurs, si un corpus de recherches plus important porte sur la formation professionnelle des adultes en général (Le Boterf, 2010, 2011; Bélanger, 2015), on observe que peu de recherches ont été menées dans le domaine de la formation relative à l'environnement auprès des adultes. Nous souhaitons contribuer à combler ces lacunes, en nous attardant plus spécifiquement à la formation d'adultes qui assument une fonction citoyenne particulière à titre d'élus à l'échelle locale ${ }^{1}$.

2 Dans cet article, nous circonscrirons d'abord la portée de la formation relative à l'environnement (FRE) par rapport à l'éducation relative à l'environnement (ERE) des adultes, pour aborder ensuite la spécificité de la FRE des élus municipaux. Puis nous exposerons les résultats d'une exploration de pratiques de formation relative à l'environnement auprès d'élus municipaux ${ }^{2}$ au Québec. Enfin, à la lumière d'une telle exploration, nous proposerons des repères pour concevoir une offre de formation qui tienne compte des diverses dimensions du rapport de ces élus à l'environnement.

\section{Les élus municipaux : des adultes aux besoins particuliers de formation}

3 Définissons d'abord sommairement l'élu municipal comme un adulte qui assume une fonction publique au sein d'un système de gouvernance démocratique, ce qui fait appel 
à des compétences professionnelles particulières. Cependant, une telle fonction diffère d'une fonction professionnelle usuelle dans la mesure où elle est dévolue au terme d'un processus électoral qui ne repose pas sur l'évaluation d'un certain corpus de compétences, mais sur le résultat d'un scrutin. Tous les adultes peuvent voter, comme tous peuvent se présenter comme candidats aux élections. Certes, cela suppose de faire preuve de certaines habiletés permettant d'être reconnu par ses concitoyens ${ }^{3}$, mais au Québec, aucun profil de compétences n'est exigé de la part des candidats (par exemple, scolarité, expérience, niveau de littératie en matière de gouvernance). La tâche est pourtant exigeante : il s'agit de représenter la population et de participer - au sein d'un Conseil municipal - à la prise de décisions sur les orientations et les priorités de la municipalité et sur l'administration des affaires collectives (MAMOT, 2016, 2018).

Les élus arrivent donc en poste avec une expérience personnelle et professionnelle très diversifiée qu'ils peuvent mobiliser pour assumer leurs fonctions politiques et qu'il importe certes de reconnaître. De même, il faut prendre en compte la variabilité des contextes politiques à l'échelle locale (par exemple, ceux des municipalités urbaines, périurbaines ou rurales) qui influence la fonction d'élu : la spécificité de chacun des contextes détermine le rôle et l'espace de pouvoir qui lui est dévolu.

5 Ici, nous nous intéressons plus spécifiquement à l'un des mandats que doit assumer l'élu au sein du Conseil : celui de la protection de l'environnement, en relation entre autres avec des lois habilitantes comme c'est le cas au Québec pour la Loi sur les compétences municipales (C-47.1) qui spécifie à l'article 4 le pouvoir des municipalités en matière d'environnement ${ }^{5}$ ou encore la Loi sur l'aménagement et l'urbanisme (c. A-19.1) qui traite de questions associées à l'environnement comme l'aménagement du territoire et la protection des cours d'eau, des milieux humides et forestiers, pour ne nommer que ceux-là'. Les municipalités québécoises doivent également se conformer à un nombre croissat de lois et règlements qui concernent l'environnement; elles doivent aussi être informées de la jurisprudence en faveur de l'environnement qui rappelle le rôle des municipalités en tant que fiduciaire de l'environnement ${ }^{7}$.

6 Il y a donc lieu de se pencher sur la formation des élus municipaux en matière d'environnement. Mais d'abord, clarifions ce qu'on entend par formation et penchonsnous sur la spécificité de l'apprenant adulte.

\section{L'éducation et la formation relative à l'environnement des adultes : un champ de pratiques encore peu théorisé}

7 La formation s'inscrit ici dans le plus vaste champ de l'éducation relative à l'environnement. Or il existe une diversité de conceptions de l'éducation relative à l'environnement qui se distinguent les unes des autres en fonction des représentations que chacune véhicule de l'environnement, de l'éducation et des relations qui les unissent au sein de divers contextes. Pour les fins de cet article, l'ERE est définie comme suit :

une dimension intégrante du développement des personnes et des groupes sociaux, qui concerne leur relation à l'environnement. Au-delà de la simple transmission de connaissances, elle privilégie la construction de savoirs collectifs dans une perspective critique. Elle vise à développer des savoir-faire utiles associés à des pouvoir-faire réels. Elle fait appel au développement d'une éthique 
environnementale et à l'adoption d'attitudes, de valeurs et de conduites imprégnées de cette éthique. (Sauvé, 1997, p. 53)

8 En suggérant cette définition, il n'est certes pas souhaité d'occulter la diversité des conceptions de l'ERE, mais bien de mettre en évidence, dans cet article, la vision globale à laquelle nous nous référons.

9 Par ailleurs, si l'ERE vise le développement des multiples dimensions de la personne (personnelles, interpersonnelles et professionnelles), la FRE concerne pour sa part, la préparation à une intervention spécifique, souvent professionnelle, en matière d'environnement (Sauvé, 1997, p.54). En Amérique du Nord, ce qui est appelé formation s'applique surtout à l'acquisition de savoirs, de savoir-faire et de savoir-être en vue du développement de certaines compétences pour agir de manière appropriée en fonction d'un contexte professionnel spécifique (Legendre, 2005, p. 622). Il est à noter que la formation découle alors d'objectifs généraux prédéterminés qui sont établis par un agent qui n'est pas l'apprenant lui-même (Sauvé, 1997, p. 50). Certains auteurs font toutefois référence à l'autoformation ou à la coformation lorsque les objectifs de formation sont choisis par l'apprenant ou par un groupe d'apprenants euxmêmes (en amont ou en cours de démarche ou d'expérience). Et lorsque c'est dans le creuset du rapport à l'environnement que s'opère la formation, de façon expérientielle, Pineau (2019) et Galvani (2020) parlent d'écoformation.

10 De plus, trois types de FRE peuvent être distingués (Sauvé, 1997, p. 55): 1) une formation générale destinée au grand public et qui suppose l'acquisition de savoirs et de savoir-faire de divers ordres en matière d'environnement (par exemple, le jardinage écologique ou le tri de matières résiduelles) ; 2) une formation spécifique destinée aux membres de certaines professions dont les tâches et les décisions sont susceptibles de concerner l'environnement (comme les ingénieurs, les urbanistes, les économistes ou les élus municipaux) ; 3) une formation spécialisée destinée aux membres des professions directement liées à l'environnement (par exemple, les spécialistes en aménagement du territoire, les écoconseillers, les éducateurs en ERE). Dans ces trois types de formation, on remarque que la FRE concerne surtout des apprenants adultes.

11 Comme souligné dans ce numéro thématique, l'ERE des adultes en général demeure un champ d'études relativement peu théorisé (Lange, 2010b ; Clover et Hill, 2013 ; Martin et Chen, 2016; Villemagne, 2008, 2017). Selon les travaux de recherche de Martin et Chen (2016), moins de trois pour cent des articles scientifiques analysés en ERE portent spécifiquement sur les adultes. La recherche en FRE des adultes apparaît tout aussi peu développée. Par ailleurs, peu de nuances sont apportées entre l'ERE et la FRE, et encore moins pour des groupes spécifiques d'adultes aux fonctions particulières.

12 Une recension d'écrits ${ }^{8}$ n'a pas permis de repérer de recherches spécifiques à la FRE des élus municipaux. Celle-ci a toutefois mené à diverses publications (par exemple, en sciences de l'environnement, en sciences politiques, en formation des adultes) qui ne traitent pas toujours directement du rapport à l'environnement, mais qui aident à éclairer certaines questions relatives à la FRE des adultes en général et qui mettent au jour certaines pratiques de formation auprès d'élus municipaux.

13 Par ailleurs, bien que la notion de pouvoir ait fait l'objet d'une attention particulière en éducation, surtout en ce qui a trait aux adultes opprimés (Freire, 1974 ; Spector, 1997), notre recension d'écrits, sans doute perfectible, a permis de constater le peu de recherches relatives à la formation d'adultes en situation de pouvoir, comme les élus 
municipaux (Kezar, 2003 ; Desmond, 2004 ; Morris, 2009 ; Rice, 2010 ; Neumann, 2011 ; Selwyn, 2012 ; Campbell, 2016).

Certains auteurs repérés ont toutefois souligné, dans leurs champs disciplinaires respectifs, l'importance de former les élus et plus spécifiquement de les interpeler sur leur rôle en matière d'environnement à l'échelle locale (MEA, 2005; Santamaría et Méndez, 2012 ; GIEC-IPCC, 2014 ; Biscotti et D'Amico, 2016; Larson et coll., 2017) ou de les former en mobilisant des approches et des stratégies d'ERE des adultes (Guertin, 2000 ; Sauvé, 2009b ; Sauvé et Girault, 2014).

\section{Regards sur la FRE offerte aux élus municipaux}

15 En réponse aux limites de la recherche existante, nous avons donc entrepris une exploration plus poussée de cet objet d'études qu'est la FRE auprès des élus municipaux. Cette exploration repose sur l'observation directe ${ }^{9}$ de telles pratiques de formation, plus spécifiquement lors de sept ateliers ou cours offerts en présentiel et de trois colloques spécialisés qui leur étaient destinés (Guertin, 2017). A également été mise à contribution ma propre pratique réflexive à titre de professionnel ou formateur en environnement œuvrant auprès d'élus à l'échelle municipale au Québec durant plus de 20 ans $^{10}$.

Pour baliser cette observation et structurer la caractérisation des pratiques, la typologie des perspectives dominantes en ERE proposée par Sauvé (1997, 2009a) a été mobilisée. Ces trois perspectives correspondent à trois façons distinctes, mais complémentaires d'aborder l'ERE. Ainsi dans une perspective socioécologique, l'ERE vise à préserver la qualité de l'environnement, support à la vie et à la qualité de vie. La perspective psychosociale de l'ERE se penche sur le développement optimal des personnes et des groupes sociaux à travers leur relation à l'environnement. Quant à la perspective pédagogique de l'ERE, elle intéresse plus spécifiquement les pédagogues qui perçoivent l'éducation relative à l'environnement comme une invitation à améliorer les conditions d'apprentissage par l'écologisation des lieux de formation et des curriculums, ainsi que par l'adoption d'une pédagogie différente des pédagogies dites traditionnelles (Sauvé, 1997, p. 23).

Nous verrons dans l'analyse qui suit que les pratiques observées en FRE des élus sont principalement associées à une perspective socioécologique et dans une moindre mesure, à une perspective psychosociale - selon la typologie que nous venons d'évoquer. Le tableau 1 présente une caractérisation sommaire de ces pratiques en FRE auprès des élus, en fonction des objets de formation privilégiés, des buts poursuivis ainsi que des approches adoptées, au regard des deux perspectives de référence. 
Tableau 1 : Caractérisation des pratiques de formation relative à l'environnement (FRE) des élus municipaux selon les perspectives socioécologique et psychosociale issues de la typologie des perspectives dominantes en ERE - Sauvé $(1997$; 2009a).

\begin{tabular}{|c|c|c|}
\hline & $\begin{array}{l}\text { Perspective socioécologique } \\
\text { (Approche dominante) }\end{array}$ & $\begin{array}{l}\text { Perspective psychosociale } \\
\text { (Approche émergeante) }\end{array}$ \\
\hline $\begin{array}{l}\text { Objets de } \\
\text { formation } \\
\text { privilégiés : } \\
\text { enjeux } \\
\text { abordés }\end{array}$ & $\begin{array}{l}\text { Les changements climatiques, la } \\
\text { gestion des matières résiduelles, les } \\
\text { eaux pluviales, la foresterie urbaine et } \\
\text { l'aménagement du territoire, en tant } \\
\text { que questions socioécologiques } \\
\text { relevant de l'autorité municipale. }\end{array}$ & $\begin{array}{l}\text { L'environnement est abordé comme } \\
\text { un milieu de vie et un projet } \\
\text { collectif. Ici, c'est le rapport } \\
\text { personnel et social à à } \\
\text { l'environnement qui est en cause. }\end{array}$ \\
\hline Buts de la FRE & $\begin{array}{l}\text { La FRE vise à doter l'élu d'un savoir- } \\
\text { agir spécifique en fonction de la } \\
\text { résolution de problèmes } \\
\text { socioécologiques et l'aménagement } \\
\text { du milieu de vie. Par exemple, } \\
\text { plusieurs formations en présentiel, des } \\
\text { webinaires et des guides sont offerts } \\
\text { sur des questions environnementales } \\
\text { spécifiques où l'on aborde les aspects } \\
\text { techniques et légaux, les systèmes de } \\
\text { gestion et les étapes de résolution de } \\
\text { problèmes spécifiques au milieu } \\
\text { municipal. }\end{array}$ & $\begin{array}{l}\text { La FRE favorise le développement de } \\
\text { compétences cognitives, réflexives, } \\
\text { éthiques et politiques permettant à } \\
\text { l'élu de mieux construire son } \\
\text { identité d'élu et de mieux } \\
\text { appréhender son rôle d'arbitre et de } \\
\text { décideur au regard des questions } \\
\text { sociales et écologiques. }\end{array}$ \\
\hline $\begin{array}{l}\text { Spécificité des } \\
\text { approches de } \\
\text { formation } \\
\text { observées }\end{array}$ & $\begin{array}{l}\text { L'accent est mis sur l'« objet " qu'est } \\
\text { l'environnement comme une réalité } \\
\text { complexe en mobilisant des approches } \\
\text { cognitives, behavioristes et } \\
\text { pragmatiques. Par exemple, les } \\
\text { formations observées reposent sur } \\
\text { l'exposé d'un expert qui aborde les } \\
\text { problématiques environnementales } \\
\text { municipales, en insistant sur les } \\
\text { moyens et l'urgence d'agir. }\end{array}$ & $\begin{array}{l}\text { L'accent est mis surtout sur l'identité } \\
\text { et le rôle social de l'élu au regard des } \\
\text { questions environnementales au } \\
\text { sein de la municipalité. } \\
\text { Les formations adoptent des } \\
\text { stratégies participatives et portent } \\
\text { attention à la signification des } \\
\text { réalités sociales et écologiques en } \\
\text { fonction du contexte où évoluent les } \\
\text { élus. L'élu comme apprenant adulte } \\
\text { est invité à participer à la recherche } \\
\text { d'information, à la réflexion critique, } \\
\text { à la discussion éthique, à la } \\
\text { recherche de de solutions } \\
\text { contextualisées, à l'élaboration d'un } \\
\text { plan d'action. }\end{array}$ \\
\hline
\end{tabular}

\section{Observations relatives à la perspective socioécologique de la FRE}

Rappelons que dans une perspective socioécologique, l'ERE aborde surtout les problèmes associés à la qualité de l'environnement, à sa dégradation, à ce qui menace la qualité de vie. L'ERE est conçue comme outil pour développer des habiletés de résolution de problèmes et d'écogestion.

19 Selon les observations que nous avons faites de situations de FRE auprès des élus municipaux, plusieurs pratiques peuvent être associées à cette perspective. La formation des élus traite ici d'enjeux comme les changements climatiques, la récupération des matières résiduelles, la gestion des eaux pluviales ou la foresterie urbaine. Elle se rattache à des domaines qui sont plus traditionnellement associés au milieu municipal (par exemple, les égouts, l'aqueduc ou l'aménagement du territoire) ou à des problèmes environnementaux aigus (par exemple, les inondations, les îlots de chaleur, la pénurie d'eau potable) qui font appel à une intervention accrue de la part des municipalités.

20 En réponse aux appels à l'action qui se multiplient de la part de panels d'experts sur la scène publique, mais également en réaction aux manifestations de plus en plus tangibles des changements climatiques au Québec (inondations, canicules, feux de forêt), on remarque que cet enjeu est largement abordé dans l'offre de formation actuelle auprès des élus. Les synopsis et les objectifs des exposés, des rencontres ou des ateliers suggèrent explicitement le développement d'un argumentaire en faveur de 
l'environnement et le développement d'habiletés visant l'adaptation aux changements climatiques à l'échelle locale.

21 Les activités de formation caractérisées par l'adoption d'une perspective socioécologique prennent la forme de webinaires, de formations en présentiel et de formations à distance. On y mobilise des approches cognitive et behaviorale, marquées par un certain pragmatisme (selon la typologie des approches proposée par Sauvé, 1997, p. 151-157). Les approches cognitives mobilisent notamment des exposés magistraux et la présentation d'études de cas pour faire connaître les stratégies d'action possibles sur des questions spécifiques. Ces activités sont parfois accompagnées de guides, de vidéos et de témoignages d'experts. Le rapport à l'environnement est aussi traité selon une approche behavioriste en mettant en évidence des stratégies comportementales pour résoudre des problèmes environnementaux: par exemple, l'écotaxation, les écofrais, les amendes, les subventions et les concours. La formation se caractérise par un certain pragmatisme, de façon à développer des habiletés de résolution de problèmes environnementaux et d'écogestion municipale. On vise à mobiliser l'appareil municipal et les élus autour de la confection de plans et le développement de projets en faveur de l'environnement.

On remarque également de manière générale que la formation offerte dans une perspective socioécologique porte souvent sur des aspects de conformité légale ou de respect des politiques relevant du gouvernement provincial ou fédéral. Elle semble ainsi peu adaptée au rôle des élus municipaux et aux outils décisionnels à leur portée dans leur espace de juridiction (par exemple les outils de planification ou financiers, les règlements et les processus décisionnels qui les accompagnent). Les élus municipaux sont des acteurs politiques dont le pouvoir d'action vise notamment à donner des orientations, à faire entre autres l'arbitrage des usages du sol, et de façon générale, à veiller au bien commun sur le territoire de leur ville (Brochu et coll., 2017). Or les contenus abordés laissent certains élus perplexes face à des tensions locales qui sont vives et difficiles à rattacher aux contenus des formations. Dans les formations offertes, le rapport au pouvoir est le plus souvent implicitement abordé sous l'angle légal et technique, plutôt qu'en le plaçant dans un contexte éthique et politique.

23 Ce constat nous porte à croire qu'une réflexion s'impose sur la formation des élus à l'égard de l'environnement: celle-ci devrait favoriser la réflexion critique sur les questions environnementales au-delà de la notion de conformité légale. Cela étant dit, il semble aussi nécessaire de réfléchir à la FRE des professionnels municipaux non élus de façon à développer chez tous les acteurs de l'appareil municipal un ensemble de compétences éthique, critique, politique et heuristique (Sauvé, 2013) au regard de questions environnementales.

24 Considérant le peu d'activités de formation consacrées principalement à l'environnement pour les élus, nous avons exploré l'offre de formation plus générale qui leur est destinée, afin d'y repérer des questions environnementales traitées de manière transversale, à travers d'autres thématiques de formation. Notamment, nous avons examiné à cet effet la formation initiale des élus souvent greffée à la formation obligatoire en éthique et déontologie municipale. 


\section{Observations relatives à la perspective psychosociale de la FRE}

Si l'on s'attarde à cette formation générale des élus municipaux, on observe que certaines pratiques peuvent être associées à une perspective psychosociale. Rappelons que les pratiques en ERE des adultes dans une perspective psychosociale - au-delà de la résolution de problèmes pragmatiques - s'intéressent plus fondamentalement à l'amélioration de la qualité de la relation entre les humains et l'environnement; notamment, il s'agit de colmater la profonde rupture entre les personnes, les sociétés et le monde vivant dont elles font pourtant partie intégrante (Sauvé, 1997, p. 22). L'ERE est ici abordée comme une dimension fondamentale de l'éducation, un processus d'apprentissage qui s'échelonne tout au long de la vie, jalonnée par une réflexion personnelle et collective sur nos rapports à l'environnement. Dans cette perspective, l'accent est mis sur le développement personnel et social vers la construction d'une identité écologique, en considérant les besoins d'appartenance et l'importance de l'engagement (Sauvé, 1997, p. 87).

Lors de la formation initiale offerte aux élus par certaines associations de municipalités au Québec, l'environnement est abordé comme un enjeu contemporain pour les villes, qui aura une incidence marquée sur la qualité de vie des citoyens. Ici, l'environnement est abordé comme une composante d'un projet collectif et doit être conjugué avec d'autres enjeux d'ordre politique, comme la mondialisation ainsi que le vieillissement de la population.

Par contre, nous avons remarqué que le rapport à l'environnement reste limité à la prise en compte d'enjeux et ne donne pas lieu à une réflexion sur le rôle des élus, leurs valeurs personnelles et leur identité politique pour œuvrer avec pertinence et cohérence à l'intérieur de l'organisation municipale au regard de l'environnement. Comme l'ont signalé Greenwood et Wilson (1995) et Paddock (1996), le rôle politique des élus est source de tension dans les municipalités; il apparait donc comme l'un des objets de formation à privilégier. Par ailleurs, les balises entre le rôle des fonctionnaires et des élus sont parfois floues dans l'esprit des nouveaux élus. On a remarqué, lors de certaines formations, une intention de clarifier cet enjeu organisationnel, de même que d'aborder le rôle de représentation des élus auprès des citoyens par l'entremise d'une réflexion personnelle et collective sur le bien commun, le « commun » comme l'explicitent Dardot et Laval (2015), de manière à éviter certaines dérives clientélistes.

De plus, l'offre actuelle de formation ne facilite pas nécessairement la réflexion sur les liens entre le développement professionnel, l'acquisition de compétences spécifiques aux fonctions d'élus et l'environnement. La formation semble se limiter au développement de compétences managériales plutôt que politiques. Or, la FRE auprès des élus apparaît pourtant comme un créneau potentiel majeur pour développer des compétences critique, éthique et politique permettant aux élus de clarifier leur identité politique et de mieux appréhender leur rôle de décideurs et d'arbitres au regard des questions relatives à l'environnement et de façon plus globale, au bien commun.

Il faut certes reconnaître, comme l'ont observé plusieurs chercheurs, que la formation et le développement professionnel des élus posent un défi de taille (Paddock, 1996). Greenwood et Wilson (1995) ainsi que Thomson (2010) constatent le peu de temps 
consacré à leur propre formation par les élus, ce qui ne facilite pas le développement de compétences fondamentales. Mais aussi, la courte durée des formations ne permet pas de prendre en compte la variabilité des contextes municipaux et par conséquent, la spécificité contextuelle du rôle des élus et de leurs expériences de vie (Paddock, 1996). Trop souvent, la formation demeure technique et n'invite pas les élus à entreprendre une réflexion en profondeur sur leur propre résistance au changement par exemple, et sur les barrières qui empêchent d'agir individuellement et collectivement.

Enfin, la formation des mairesses et des maires exige une attention particulière : les compétences requises diffèrent de celles des conseillères et conseillers (Karsten, 2019). Entre autres, comme l'ont montré Mévellec et Tremblay (2016), ils n'ont certainement pas les mêmes motivations, notamment si l'on aborde la formation au regard des questions d'identité et de genre (p. 66).

\section{Repères pour la recherche et la pratique en matière de FRE auprès des élus municipaux}

31 L'observation des pratiques de formation des élus montre qu'au-delà des limites actuelles, la convergence des perspectives socioécologique et psychosociale de l'ERE/ FRE peut offrir un riche terreau pour concevoir une offre de formation adaptée aux élus municipaux. À cet effet, à la lumière de fondements en matière d'éducation et formation relatives à l'environnement des adultes, et mettant à profit certains éléments de pratique observés, il est possible de suggérer des repères pour une formation appropriée.

Une telle préoccupation de développement pédagogique (ici, andragogique) s'inscrit dans la troisième perspective selon laquelle il est possible d'aborder l'ERE/FRE - telle que décrite par Sauvé, 2009 - et qui a pour visée l'amélioration de la dynamique de formation elle-même. Il s'agit d'assurer la pertinence et la complétude des objectifs et des contenus des programmes de formation au regard de l'ensemble des dimensions de la relation à l'environnement, d'adopter des stratégies de formation appropriées et aussi d'écologiser les lieux de formation, en mettant l'accent sur l'environnement comme un creuset du rapport à l'environnement où s'opère une écoformation.

33 L'élaboration de tels repères fait l'objet d'une thèse de doctorat en préparation ${ }^{11}$. Cependant les premiers travaux que nous avons menés permettent d'identifier déjà certaines balises :

- Tout en reconnaissant leur pouvoir politique, les élus municipaux doivent être abordés comme des apprenants adultes aux besoins particuliers. Cela oblige à la prise en compte des principes andragogiques de base (Knowles, 1990 ; Panneton, 1994 ; Boucouvalas et LipsonLawrence, 2010). En particulier, le principe qui stipule que l'apprenant adulte se trouve au centre de son apprentissage et que l'apprentissage est souvent animé par un besoin précis (par exemple, une tâche à accomplir ou un problème concret). Autre principe important, il importe de reconnaitre le vécu expérientiel de l'adulte et de considérer le groupe (regroupement d'élus, le conseil municipal, l'organisation municipale, ses concitoyens) comme une ressource pour l'apprentissage. Il est aussi nécessaire de reconnaitre le principe andragogique qui stipule que le processus d'apprentissage chez les adultes est une démarche autant affective que cognitive et qu'il importe de se préoccuper de manière globale de son développement professionnel et personnel. 
- Il ne s'agit donc pas d'enfermer la FRE auprès des élus dans une conception étroite de l'ERE ou même de la formation continue, mais bien de favoriser chez les formateurs une clarification de leurs pratiques de formation et de les inciter à associer les choix andragogiques à une démarche critique en ce qui concerne le rapport à l'environnement. Une telle formation reconnait les avantages, mais aussi les limites de choix contextuellement adaptés, et qui les articule de façon cohérente, en fonction d'un référentiel explicite et justifié.

- De manière plus pratique, il semble nécessaire d'aborder la FRE auprès des élus comme un élément essentiel de leur formation continue. Les activités de formation offertes et les questions abordées doivent à la fois situer l'environnement comme une compétence municipale (au sens politique), mais aussi permettre d'y réfléchir de manière heuristique, c'est-à-dite de façon à envisager d'autres façons d'aborder les réalités socioécologiques et de concevoir des projets. Il importe aussi de cadrer les activités de formation par rapport à la diversité des contextes municipaux. On aborde le «monde municipal » parfois comme un tout homogène sans considérer son caractère urbain ou rural, ni l'incidence de la taille des organisations municipales sur les rôles des élus. Il importera alors de faire référence à une diversité de cas et de situations réelles de manière à rendre compte de la pluralité des contextes et des divers rôles que doivent assumer les élus. De plus, il semble approprié de mobiliser des approches expérientielles, collaboratives et interdisciplinaires ainsi que des stratégies participatives; de telles approches favorisent la prise en compte de la complexité des rapports à l'environnement à l'échelle municipale et contribuent au développement de compétences écocitoyennes (Sauvé, 2013 ; Pruneau et coll., 2017).

- Bien que nos observations reposent sur des pratiques menées dans un contexte non formel structuré par des associations de municipalités, il semble opportun aussi de considérer la FRE dans un contexte informel, c'est-à-dire, un contexte de formation non systématique, non rattaché à aucune structure particulière et qui ne fait pas l'objet d'activités planifiées dans le cadre d'un programme ou d'une évaluation finale (Flowers et coll., 2009). Les élus arrivent en formation structurée avec une riche expérience et des connaissances acquises dans le cadre de leurs fonctions d'élus, de même que des savoirs provenant d'autres acteurs municipaux, des ONG, divers médias ainsi que le milieu de la recherche. Les élus arrivent avec une intention, un besoin. Il importerait alors de reconnaître leur démarche d'autoformation ou de coformation, où les apprentissages se font individuellement, de manière autodirigée ou fortuite, au gré des échanges avec des citoyens, d'autres élus, des professionnels municipaux et des experts dans divers domaines de la gestion municipale (Tough, 2002 ; Tremblay, 2003 ; Campbell-Smeltzer, 2016).

34 Ainsi le travail reste à poursuivre pour améliorer la formation des élus municipaux en matière d'environnement. Par exemple, dès le départ, une plus grande attention devrait être accordée aux représentations des élus à l'égard de l'environnement, des compétences à développer et des modes de formation à privilégier. Il en va de même pour la clarification des représentations des formateurs d'élus.

Les repères que nous proposons pour la FRE auprès des élus municipaux restent à préciser, à discuter et à passer au test de la pratique avec des acteurs d'une telle formation, soit les formateurs et les élus. Il semble toutefois d'entrée de jeu que ces repères offrent des balises pour mobiliser divers champs de savoirs et pour déployer une FRE ancrée dans la diversité des milieux municipaux. Il s'agit de contribuer au développement d'élus écocitoyens, capables d'excercer un leadership critique, engagé, 
compétent et apte à participer aux débats publics et à la transformation des politiques et pratiques écosociales (Sauvé, 2016).

\section{Conclusion}

Dans cet article, nous avons caractérisé sommairement l'offre de formation des élus dans un contexte non formel structuré par diverses associations de municipalités, en collaboration avec divers ministères québécois et organisations non gouvernementales. Nous avons mobilisé à cet effet une typologie de perspectives de formation, initialement développée dans le domaine de l'ERE: les perspectives socioécologique, psychosociale et pédagogique.

Nous avons ensuite proposé quelques repères pour la conception de programmes ou d'initiatives de formation appropriée aux élus municipaux. De façon générale, il nous semble essentiel de reconnaitre les besoins spécifiques des élus en considérant les principes généraux d'andragogie. De plus, il nous parait nécessaire d'aborder la formation d'une manière critique en clarifiant les pratiques en fonction d'un référentiel explicite et justifié. Il serait aussi pertinent d'élargir le spectre d'analyse de l'offre de formation en mettant l'accent sur la finalité, les prémisses, les objectifs, les contenus et la place du sujet apprenant dans la démarche de formation. Enfin, puisqu'on s'intéresse aux décideurs locaux, il semble important de reconnaitre la diversité des contextes municipaux et leur incidence sur le rôle politique des élus au regard de l'environnement. Selon le contexte, la FRE fera appel à des stratégies qui répondent parfois à la perspective socioécologique, de manière à résoudre des questions urgentes, à la perspective psychosociale, de manière à développer des compétences nécessaires pour répondre aux besoins des élus ou à la perspective pédagogique, de manière à favoriser l'apprentissage autant " pour ", qu' « au sujet » de l'environnement et « dans » celui-ci.

Enfin, il serait souhaitable de valider et de documenter davantage les pratiques de FRE auprès des élus à la lumière entre autres des théories du domaine de l'andragogie. Il semble qu'un cadre collaboratif de recherche se prêterait bien à un tel exercice. Les étapes à venir de notre projet de recherche-développement visent notamment à établir de telles collaborations pour favoriser l'optimalisation de la formation relative à l'environnement auprès des élus municipaux.

\section{BIBLIOGRAPHIE}

Bélanger, P. (2015). Parcours éducatifs, construction de soi et transformation sociale. Québec: Presses de l'Université du Québec.

Biscotti, A. M. et D'Amico, E. (2016). What are political leaders' environmental intentions? The impact of social identification processes and macro-economic conditions. Ecological Economics, 129, 152-160. 
Boucouvalas, M. et Lipson-Lawrence, R. (2010). Adulte learning. Dans C. E. Kasworm, A. D. Rose et J. M. Ross-Gordon (dir.), Handbook of Adult and Continuing Education - 2010 Edition (p. 35-48). NewYork : SAGE Publications.

Brochu, E. (coord.), M. (2017). Guide d'accueil et de référence pour les élus municipaux 2017. Québec : Gouvernement du Québec, ministère des Affaires municipales et de l'Occupation du territoire.

Campbell, S. D. (2016). The Planner's Triangle Revisited : Sustainability and the Evolution of a Planning Ideal That Can't Stand Still. Journal of the American Planning Association, 82(4), 388-397.

Campbell- Smeltzer, R. C. (2016). The Learning Projects of Municipal Elected Officials. Thèse de doctorat inédite. University of Tennessee - Knoxville. Récupéré sur https://trace.tennessee.edu/ utk_graddiss/3746/

Clover, D. E. et Hill, R. (2013). Adult Learning, Education, and the Environment. New Directions for Adult and Continuing Education, Été (138), 49-59.

Dardot, P. et Laval, C. (2015). Commun. Essai sur la révolution au XXIe siècle. Paris : La Découverte. Desmond, M. (2004). Methodological challenges posed an elite in the field. Area, 36(3), 262-269.

Flowers, R., Guevara, R. et Whelan, J. (2009). Popular and informal environmental education - The need for more research in an "emerging" field of practice. Rapport de recherche (2/2009/32.Jg.). Bonn : German Institut for Adult Education / Deutsches Institut für Erwachsenenbildung.

Freire, P. (1974). Pédagogie des opprimés et Conscience et révolution, traduits du brésilien par François Maspero. Paris : Librairie François Maspero.

Galvani, P. (2020). Autoformation et connaissance de soi. Lyon : Chronique sociale.

GIEC-IPCC. (2014). Changements climatiques 2014 : 5e rapport de synthèse - résumé à l'intention des décideurs. Genève. Récupéré sur https://archive.ipcc.ch/pdf/assessment-report/ar5/syr/ SYR_AR5_FINAL_full_fr.pdf

Greenwood, J. et Wilson, D. (1995). Councillor effectiveness : What role for training ? Local Government Studies, 21(3), 432-447.

Guertin, M.-A. (2000). Diagnostic des représentations de l'environnement chez les décideurs dans une perspective de formation relative à l'environnement : le cas de la protection du site de nidification du faucon pèlerin de la Réserve de la biosphère (UNESCO) du mont Saint-Hilaire, Québec. Mémoire de maîtrise inédit. Université du Québec à Montréal.

Guertin, M.-A. (2017). Vers des stratégies de formation des élus municipaux aux enjeux socio-écologiques et à l'aménagement du territoire - projet de thèse. Université du Québec à Montréal (UQAM) Programme de doctorat en sciences de l'environnement.

Karsten, N. (2019). What Councillors Expect of Facilitative Mayors : The Desired Leadership Competencies in Job Advertisements for the Dutch Mayoralty and How They Are Affected by Municipal Size. Lex localis - Journal of Local Self-Government, 17(1), 179-199.

Kezar, A. (2003). Transformative Elite Interviews : Principles and Problems. Qualitative Inquiry, 9(3), 395-415.

Knowles, M. S. (1990). L'apprenant adulte : vers un nouvel art de la formation. Paris : Éditions d'Organisation.

Lange, E. A. (2010a). Environmental Adult Education : A Many-Voiced Landscape. Dans C. E. Kasworn, A. D. Rose et J. M. Ross-Gordon (dir.), Handbook of Adult and Continuing Education - 2010 Edition (chap. 28, p. 305-315). Los Angeles : SAGE Publications. 
Lange, E. A. (2010b). Environmental Adult Education : A Many-Voiced Landscape. Dans C. E. Kasworn, A. D. Rose et J. M. Ross-Gordon (dir.), Handbook of Adult and Continuing Education - 2010 Edition (p. 305-315). New-York : SAGE Publications.

Larson, L. R., Lauber, T. B., Kay, D. L. et Cutts, B. B. (2017). Local Government Capacity to Respond to Environmental Change : Insights from Towns in New York State. Environmental Management, 60(1), 118-135.

Le Boterf, G. (2010). Professionnaliser - Construire des parcours personnalisés de professionnalisation. Paris : Eyrolles - Édition d'Organisation, $6^{\mathrm{e}}$ édition.

Le Boterf, G. (2011). Qu'est-ce qu'un professionnel compétent ? Comment développer son professionnalisme ? Pédagogie collégiale, 24(2), 27-31.

Legendre, R. (2005). Dictionnaire actuel de l'éducation. Montréal : Guérin, 3e édition

MAMOT. (2016). L'organisation municipale au Québec en 2016. Ministère des Affaires municipales et de l'Organisation du territoire (MAMOT) - Gouvernement du Québec. Récupéré sur

https://www.mamh.gouv.qc.ca/fileadmin/publications/organisation_municipale/ organisation_territoriale/organisation_municipale_2016.pdf

MAMOT. (2018). Organisation territoriale - Quel est le rôle du conseil municipal et des élus ? ministère des Affaires municipales et de l'Occupation du territoire (МАMOT) - Gouvernement du Québec. Récupéré sur https://www.mamh.gouv.qc.ca/organisation-municipale/democratie-municipale/ conseil-municipal-et-elus/role-du-conseil-et-des-elus/

Martin, A. R. et Chen, J. C. (2016). Barriers to sustainability in mature-age adult learners : working toward identity change. Environmental Education Research, 22(6), 849-867.

MEA, M. (2005). Current state and trends assessment - Millenium Ecosystem Assessment. Washington, D.C. : Island Press.

Mévellec, A. et Tremblay, M. (2016). Genre et professionnalisation de la politique municipale - Un portrait des élues et élus du Québec. Québec : Presses de l'Université du Québec.

Morris, Z. S. (2009). The truth about interviewing elites. Politics, 29(3), 209-217.

Neumann, E. (2011). Negotiating Power : Interviews with the Policy Elite--Stories from Hungary Lost between Genres. European Educational Research Journal, 10(2), 225-232.

Paddock, S. (1996). Training municipal elected officials : Current practice and a developmental model. International Journal of Public Administration, 19(5), 689-709.

Panneton, F. (1994). La formation relative à l'environnement : design d'un module de formation en gestion environnementale intégrée à l'intention des décideurs de la PME. Rapport de recherche présenté comme exigence partielle de la maîtrise en sciences de l'environnement. Mémoire de maîtrise inédit. Université du Québec à Montréal.

Pineau, G. (2019). Cinq ouvrages récents de la voie buissonnière écoformatrice. Éducation relative à l'environnement : Regards - Recherches - Réflexions, 15(1). 1-5.

Pruneau, D., Kerry, J. et Langis, J. (2017). Compétences citoyennes pour des communautés écologiquement viables - chapitre 10. Dans Éducation, environnement, écocitoyenneté - repères contemporains (p. 177-191). Québec : Presses de l'Université du Québec.

Rice, G. (2010). Reflections on interviewing elites. Area, 42(1), 70-75.

Santamaría, L. et Méndez, P. F. (2012). Evolution in biodiversity policy - current gaps and future needs. Evolutionary Applications, 5(2), 202-218. 
Sauvé, L. (1997). Pour une éducation relative à l'environnement - élément de design pédagogique, Guide de développement professionnel à l'intention des éducateurs. Montréal : Éditions Guérin - Eska, $2^{\mathrm{e}}$ édition.

Sauvé, L. (2009a). Being Here Together. Dans M. McKenzie, P. Hart, B. Heesoon et B. Jickling (dir.), Fields of Green : Re-Storying Culture, Environment, and Education (p. 325-335). New Jersey : Hampton Press.

Sauvé, L. (2009b). Le rapport entre éthique et politique : un enjeu pour l'éducation relative à l'environnement. Éducation relative à l'environnement : Regards - Recherche - Réflexions, 8, 147-162.

Sauvé, L. (2013). Au cœur des questions socio-écologiques : des savoirs à construire, des compétences à développer. Éducation relative à l'environnement - Regards, Recherches, Réflexions, 11, 19-40.

Sauvé, L. (2016). L'engagement écologique au cœur de la cité. Relations, 2016(786), 4.

Sauvé, L. et Girault, Y. (2014). Les enjeux éthiques des politiques publiques en matière d'environnement. Revue internationale d'éthique sociétale et gouvernementale, 16(1).

Selwyn, N. (2012). Researching the Once-Powerful in Education : the Value of Retrospective Elite Interviewing in Education Policy Research. Journal of Education Policy, 28(3), 339-352.

Spector, C. (1997). Le pouvoir. Paris : Flammarion.

Thomson, D. E. (2010). The evolving role of universities in expanding knowledge among local elected officials. Journal of Public Affairs Education, 16(3), 421-450.

Tough, A. (2002). The Iceberg of Informal Adult Learning. Résumé de conférence communication présentée au The research Network for New Approaches to Lifelong Learning (NALL), Toronto. Récupéré sur https://nall.oise.utoronto.ca/res/49AllenTough.pdf

Tremblay, N. A. (2003). L'autoformation : Pour apprendre autrement. Montréal : Presses de l'Université de Montréal.

Van Zanten, A. (2013). Ethnographie. Dans Les 100 mots de la sociologie (p. 33-34). Paris : Presse universitaire de France.

Villemagne, C. (2008). Regard sur l' éducation relative à l' environnement des adultes. Vertigo revue électronique en sciences de l'environnnement, 8(1), 1-16.

Villemagne, C. (2017). Éducation relative à l'environnement des adultes. Dans L. Sauvé, I. Orellana, C. Villemagne et B. Bader (dir.), Éducation, environnement et écocitoyenneté - repères contemporains (chap. 9, p. 157-176). Québec : Presse de l'Université du Québec.

\section{NOTES}

1. L'analyse que nous présentons dans cet article fait partie d'un plus vaste projet de recherche doctorale en cours (Institut des sciences de l'environnement, UQAM) visant le développement de propositions théoriques et pratiques concernant la formation relative à l'environnement des élus municipaux.

2. Tout en reconnaissant la diversité des représentations du rôle des élus municipaux en fonction du genre, le terme élu sera utilisé pour alléger le texte et représente autant les femmes et les hommes qui assument la fonction de mairesse ou de maire ou celle de conseillère ou conseiller au sein d'une administration municipale au Québec.

3. Précisons que pour être élu au Québec, il faut avoir 18 ans, ne pas avoir été reconnu coupable d'actes frauduleux et d'être assujetti à une curatelle. Ensuite s'ajoutent des critères 
administratifs associés au lieu de résidence et au statut de citoyen ainsi qu'au cumul de fonctions qui peuvent rendre inéligible un candidat à une élection. Pour être élu, le candidat devra ultimement remporter la majorité des votes au suffrage universel. (https:// www.presentezvous.gouv.qc.ca/je-pose-ma-candidature/qui-peut-se-presenter-aux-electionsmunicipales/)

4. Pour un aperçu de la diversité des contextes municipaux au Québec, on peut consulter MAMH (2018 et 2019).

5. La Loi sur les compétences municipales (C-47.1) spécifie le pouvoir des municipalités en matière d'environnement au paragraphe 4 de l'article 4 : «En outre des compétences qui lui sont conférées par d'autres lois, toute municipalité locale a compétence dans les domaines suivants : $4^{\circ}$ l'environnement » (http://legisquebec.gouv.qc.ca/fr/showdoc/cs/C-47.1).

6. La Loi sur l'aménagement et l'urbanisme - LAU - (LRQ, c. A-19.1) est la principale loi provinciale régissant l'aménagement et l'organisation du territoire au Québec. Cette loi permet d'identifier des zones d'intérêt aux fins de protections de l'environnement comme les cours d'eau, les plaines inondables, les milieux humides et forestiers. En plus de favoriser la protection et la mise en valeur des milieux naturels, la LAU assure aussi les conditions de sécurité et de salubrité publique associées aux risques naturels et technologiques ainsi qu'à la pollution de tout genre (http:// legisquebec.gouv.qc.ca/fr/ShowDoc/cs/A-19.1).

7. Parmi les lois provinciales en matière d'environnement auxquelles sont assujetties les municipalités, on note la Loi sur la qualité de l'environnement (LQE - Q-2) qui compte plus d'une cinquantaine de règlements d'application, la Loi affirmant le caractère collectif des ressources en eau et favorisant une meilleure gouvernance de l'eau et des milieux associés (LCCE- C-6.2), la Loi concernant la conservation des milieux humides et hydriques (LCMHH - C-14), la Loi sur la conservation du patrimoine naturel (LCPN - C-61.01) auxquelles il faut ajouter une importante jurisprudence qui rappelle le rôle de fiduciaire de l'environnement des municipalités au Québec (Affaire Spraytech c. Hudson [2001], Corporation municipale du Canton de Hatley c. Développement Bacon's Bay inc.[1993], Ville de Barkmere c. Hardie [1977], 9034-8822 Québec inc. c. Ville de Sutton [2010], Frelighsburg c. Sibeca [2004], Wallot c. Ville de Québec [2011]).

8. La recension visait à explorer les écrits relatifs à la formation relative à l'environnement (FRE) et plus spécifiquement la FRE auprès des élus municipaux. La recension a été menée à l'aide des bases de données ERIC et SCOPUS qui regroupent des articles portant sur l'éducation et l'environnement. Les mots clés " formation » et « élu » ont été déclinés pour identifier un large spectre d'écrits : éducation, formation, éducation relative à l'environnement (ERE) et FRE, formation continue, formation professionnelle et formation tout au long de la vie; adultes, décideurs, élu, maire et conseillers. Des synonymes en anglais ont aussi été utilisés ainsi que les formes au féminin et au pluriel. La sélection des articles a reposé sur les critères suivants : la concordance du contenu au regard des thèmes associés au projet de recherche; la couverture des plus récents textes et ouvrages : de 1990 à 2017, la fiabilité de la source en privilégiant les périodiques scientifiques revus par des pairs, ensuite les livres et chapitres de livres, puis les thèses et mémoires. 119 écrits ont été repérés, 90 résumés ont été lus et 30 écrits ont fait l'objet d'une analyse plus systématique pour identifier les objets d'intérêt de la formation dont l'environnement, les buts, les relations entre le formateur et les apprenants, les approches ainsi que les tensions et enjeux associés à ces processus de formation.

9. Bien que plusieurs auteurs constatent la constante mouvance entourant les pratiques ethnographiques et l'absence de définitions consensuelle, l'observation directe peut être sommairement décrite comme une approche d'analyse du social et de la culture qui se caractérise par un rapport prolongé au «terrain » ou à l'observation des participants dans leur milieu de vie (Van Zanten, 2013).

10. Il s'agit donc de ma propre expérience, en tant qu'auteur de cet article. Ce texte est rédigé au «nous » de façon à reconnaître mon ancrage dans une communauté de recherche. 
11. Les objectifs généraux de cette recherche sont les suivants: 1) Mettre au jour les besoins de formation des élus municipaux au Québec en matière d'environnement de même que les enjeux associés à une telle formation. 2) Proposer les principales caractéristiques d'un processus approprié de formation relative à l'environnement auprès d'élus municipaux.

\section{RÉSUMÉS}

Cet article présente les résultats d'une caractérisation des pratiques de formation relative à l'environnement auprès d'élus municipaux, à la lumière de fondements théoriques de l'éducation relative à l'environnement et de la formation professionnelle des adultes. L'analyse a porté sur un ensemble d'activités de formation qu'il a été possible d'observer au Québec. Elle a aussi été alimentée par ma propre pratique réflexive tout au long de mon expérience de 20 ans d'accompagnement professionnel en matière d'environnement en contexte municipal. L'exercice de caractérisation s'attarde plus spécifiquement aux contenus de la formation, aux buts poursuivis et aux approches adoptées. Ces aspects sont interprétés au regard de deux perspectives de formation distinctes : une perspective socioécologique, largement dominante, et une perspective psychosociale en émergence. Nous verrons qu'au-delà des limites actuelles, la convergence de ces deux perspectives peut offrir un riche terreau pour concevoir une offre de formation adéquate, bien adaptée aux élus municipaux. Nous proposons des repères à cet effet.

This article presents the results of a characterization of environmental training practices carried out for elected municipal officials, in the light of the theoretical foundations of environmental education and continuing education for adults. The analysis focused on training activities observed in Quebec's (Canada) municipal context. It was also mediated by my own reflective practice as an environmental professional working in a municipal context over a span of 20 years. The characterization focused more specifically on the contents of the training, the goals pursued, and the approaches adopted. These aspects are interpreted in the light of two distinct training perspectives: a socio-ecological perspective, largely dominant, and an emerging psychosocial perspective. We will see that beyond the current limits, the convergence of these two perspectives can provide a rich breeding ground for the design of training programs suited for elected municipal officials. We offer benchmarks for this purpose.

\section{INDEX}

Keywords : public decision makers, municipalities, adult learners, continuing education, nonformal, informal, environmental training

Mots-clés : décideurs publics, municipalités, apprenants adultes, formation continue, contexte non formel et informel, formation relative à l'environnement

Index géographique : Québec 


\section{AUTEUR}

\section{MARC-ANDRÉ GUERTIN}

Marc-André Guertin est doctorant en sciences de l'environnement à l'Université du Québec à Montréal (UQAM). Il détient une formation de 2e cycle en éducation relative à l'environnement de l'UQAM et de 3e cycle en pédagogie de l'enseignement supérieur de l'Université de Sherbrooke (Udes). Il enseigne au Centre universitaire de formation en environnement et développement durable de l'UdeS. De plus, il possède une expérience appréciable en conseil stratégique en environnement à l'échelle municipale. [Courriel : Marc-Andre.Guertin @ USherbrooke.ca] 